International Journal of Current Microbiology and Applied Sciences

ISSN: 2319-7706 Volume 6 Number 8 (2017) pp. 3256-3259

Journal homepage: http://www.ijcmas.com

Original Research Article

https://doi.org/10.20546/ijcmas.2017.608.388

\title{
Efficacy of Selected Insecticides against Sucking Insect Pests [Amrasca biguttula biguttula (Ishida) and Bemisia tabaci (Gennadius)] of Okra [Abelmoschus esculentus (L.) Moench]
}

\author{
K.N. Prem Kumar and Ashwani Kumar* \\ Department of Entomology, Sam Higginbottom University of Agriculture, Technology \& \\ Sciences, Allahabad-211007(U.P.), India \\ *Corresponding author
}

\begin{tabular}{|c|c|}
\hline & A B S T R A C T \\
\hline $\begin{array}{l}\text { Okra, Amrasca } \\
\text { biguttula biguttula, } \\
\text { Bemesia tabaci, } \\
\text { Insecticides. }\end{array}$ & \multirow{3}{*}{$\begin{array}{l}\text { The field studies were conducted during kharif } 2016 \text { to determine the } \\
\text { efficacy of selected insecticides against sucking insect pests Amrasca } \\
\text { biguttula biguttula (Ishida) and Bemesia tabaci (Gennadius) of okra at } \\
\text { Central agriculture field, SHUATS (Sam Higginbottom University of } \\
\text { Agriculture, Technology and Sciences), Allahabad, Uttar Pradesh (India). } \\
\text { Studies revealed that all the treatments were found effective in reducing the } \\
\text { population of whitefly and jassid as compared to control, Imidiacloprid } \\
17.8 \mathrm{SL} \text { was the most effective treatment indicating reduction in population } \\
\text { of whitefly (1.33/3leaves) and jassid (1.26/3leaves), followed by } \\
\text { Thiamethoxam } 25 \mathrm{WG.}\end{array}$} \\
\hline Article Info & \\
\hline $\begin{array}{l}\text { Accepted: } \\
\text { 26 June } 2017 \\
\text { Available Online: } \\
\text { 10 August } 2017\end{array}$ & \\
\hline
\end{tabular}

\section{Introduction}

Okra [Abelmoschus esculentus (L.) Moench] is most popular vegetable of the family Malvaceae. In India, it is grown both in summer and rainy seasons (Lal and Sinha, 2005). Tender fruits of okra are used as vegetable or in culinary preparations as sliced and dried pieces. It is also used for thickening gravies and soups, because of its high mucilage content. They are also good source, vitamins, proteins, carbohydrates, minerals, iron, calcium, potassium and acids viz., rhamnose $(22 \%)$, galacturonic acid $(27 \%)$ and amino acid (11\%). It is an important vegetable crop cultivated all over India with a major share in state of Maharastra, West Bengal, Uttarpradesh, Karnataka, Gujrat and Madhya Pradesh (Shinde et al., 2007).
One of the important limiting factors in the cultivation of okra is insect pests. Many of the pests occurring on cotton are found to ravage okra crop. As high as 72 species of insects have been recorded on okra (Srinivasa and Rajendran, 2003), of which, the sucking pests comprising of Aphids, Aphis gossypii (Glover), leafhopper, Amrasca biguttula biguttula (Ishida) and whitefly, Bemisia tabaci (Gennadius) causes significant damage to the crop.

Among different sucking insect pests, jassid and whitefly are more serious (Atwal, 1994) and transmit certain viral diseases. Moreover, they cause a great damage by sucking the plant sap. Pests cause $35-40 \%$ crop yield 
losses and ultimately increase the level of damage up to $60-70 \%$ in optimal conditions (Salim, 1999).

Jassids both nymphs and adults suck the cell sap usually from the ventral surface of the leaves and while feeding inject toxic saliva into plant tissues, affected leaves turn yellowish and curl Whitefly are the milky white minute flies; nymphs and adults suck the cell sap from the leaves. The affected leaves are curled and dried. The affected plants show a stunted growth. Whiteflies are also responsible for transmitting yellow vein mosaic virus (Singh et al., 2008).

\section{Materials and Methods}

The experiment was conducted during the kharif season 2016 at Central field, SHUATS, Allahabad. The okra seeds of variety BND777 were sown by dibbling method with spacing of $45 \mathrm{~cm} \times 30 \mathrm{~cm}$ by placing 2-3 seeds per hill. Gap filling and thinning was done to maintaining the optimum plant density and prevents competition among the plants.

The experiment was laid out in randomized block design with eight treatments and three replication. The observations on the number of leafhoppers and whiteflies were made, a day before followed by 3rd, 7th, 14th days after spraying and data were recorded from three leaves each from top, middle and bottom leaves from the five randomly selected and tagged plants from each plot without disturbing the plants to minimize the observational errors. Population of sucking pest was recorded from each net plot and the population was worked out per three leaves. The data were subjected to statistical analysis.

\section{Results and Discussion}

The results of the study on the efficacy of selected insecticides against whiteflies and jassid population are presented in Table 1 and Table 2 respectively.

\section{Whiteflies}

The overall mean result represented in the table reveals that all the treatments were significantly superior over control. Among all the treatments Imidacloprid (1.33 whiteflies/3leaves) is most effective and recorded lowest population followed by Thiamethoxam (1.46 whiteflies/3leaves), followed by Fipronil (2.06 whiteflies/3leaves) and Dimethoate (2.18 whiteflies/3leaves) are at par with eachother, next treatment followed by lambdacyhalothrin (3.03 whiteflies/3leaves), Malathion (3.50 whiteflies/3leaves), while Azadirachtin (4.10 whiteflies/3leaves) is least effective among all the treatments.

These findings are in accordance with the findings of Begum and patil (2016) reported that imidacloprid $17.8 \mathrm{SL}$ was the most effiective treatment indicating reduction in population of leafhoppers and whiteflies Similarly, Pawar et al., (2016) reported that, mean population of aphids, jassids, and whiteflies after three sprays revealed that imidacloprid was effective and superior, the next best were thiomethoxam. Preetha et al., (2009) reported that imidacloprid was found effective against jassids and whiteflies, the other neonicotinoid, thiamethoxam also provided similar levels of protection as that of imidacloprid.

Sarkar (2016) performance of insecticides against whitefly was recorded in imidacloprid treated plots with lowest mean population.

\section{Jassid}

The result represented in the table reveals that all the treatments were significantly superior over control. 
Int.J.Curr.Microbiol.App.Sci (2017) 6(8): 3256-3259

Table.1 Efficacy of selected insecticides on Whitefly in okra

\begin{tabular}{|c|c|c|c|c|c|c|c|c|c|c|}
\hline \multirow{3}{*}{$\begin{array}{l}\text { Sl. } \\
\text { No. }\end{array}$} & \multirow{3}{*}{ Treatments } & \multicolumn{8}{|c|}{ Number of whiteflies/3leaves } & \multirow{3}{*}{$\begin{array}{c}\text { Overall } \\
\text { Mean }\end{array}$} \\
\hline & & \multicolumn{4}{|c|}{ First spray } & \multicolumn{4}{|c|}{ Second spray } & \\
\hline & & 1 DBS & 3 DAS & 7 DAS & 14 DAS & 1 DBS & 3 DAS & 7 DAS & 14 DAS & \\
\hline 1 & $\begin{array}{c}\text { Thiamethoxam } \\
\text { 25\%WG }\end{array}$ & $\begin{array}{l}7.53 \\
(2.74)\end{array}$ & $\begin{array}{c}1.53 \\
(1.23)\end{array}$ & $\begin{array}{c}1.26 \\
(1.12)\end{array}$ & $\begin{array}{l}1.93 \\
(1.39)\end{array}$ & $\begin{array}{c}5.73 \\
(2.36)\end{array}$ & $\begin{array}{c}1.40 \\
(1.17)\end{array}$ & $\begin{array}{l}1.13 \\
(1.06)\end{array}$ & $\begin{array}{l}1.53 \\
(1.23)\end{array}$ & $\begin{array}{l}1.46 \\
(1.21)\end{array}$ \\
\hline 2 & $\begin{array}{c}\text { Azadiractin } \\
5 \% \mathrm{EC}\end{array}$ & $\begin{array}{c}7.46 \\
(2.73)\end{array}$ & $\begin{array}{c}3.54 \\
(1.88)\end{array}$ & $\begin{array}{c}3.13 \\
(1.76)\end{array}$ & $\begin{array}{c}4.54 \\
(2.12)\end{array}$ & $\begin{array}{c}8.67 \\
(2.93)\end{array}$ & $\begin{array}{c}4.34 \\
(2.08)\end{array}$ & $\begin{array}{c}4.13 \\
(2.03)\end{array}$ & $\begin{array}{c}4.94 \\
(2.22)\end{array}$ & $\begin{array}{l}4.10 \\
(2.02)\end{array}$ \\
\hline 3 & $\begin{array}{c}\text { Imidacloprid } \\
17.8 \% \text { SL }\end{array}$ & $\begin{array}{c}7.66 \\
(2.76)\end{array}$ & $\begin{array}{c}1.46 \\
(1.21)\end{array}$ & $\begin{array}{c}1.13 \\
(1.06)\end{array}$ & $\begin{array}{l}1.73 \\
(1.31)\end{array}$ & $\begin{array}{c}5.86 \\
(2.40)\end{array}$ & $\begin{array}{c}1.33 \\
(1.15)\end{array}$ & $\begin{array}{c}1.06 \\
(1.03)\end{array}$ & $\begin{array}{c}1.26 \\
(1.12)\end{array}$ & $\begin{array}{l}1.33 \\
(1.15)\end{array}$ \\
\hline 4 & Fipronil 5\% SC & $\begin{array}{c}7.93 \\
(2.81)\end{array}$ & $\begin{array}{c}1.94 \\
(1.39)\end{array}$ & $\begin{array}{c}1.66 \\
(1.29)\end{array}$ & $\begin{array}{c}2.74 \\
(1.65)\end{array}$ & $\begin{array}{c}6.53 \\
(2.52)\end{array}$ & $\begin{array}{c}2.06 \\
(1.43)\end{array}$ & $\begin{array}{c}1.67 \\
(1.29)\end{array}$ & $\begin{array}{c}2.34 \\
(1.52)\end{array}$ & $\begin{array}{c}2.06 \\
(1.43)\end{array}$ \\
\hline 5 & Dimethoate 30EC & $\begin{array}{c}7.74 \\
(2.77)\end{array}$ & $\begin{array}{c}2.15 \\
(1.43)\end{array}$ & $\begin{array}{c}1.76 \\
(1.31)\end{array}$ & $\begin{array}{c}2.66 \\
(1.63)\end{array}$ & $\begin{array}{c}6.93 \\
(2.62)\end{array}$ & $\begin{array}{c}2.13 \\
(1.45)\end{array}$ & $\begin{array}{c}1.86 \\
(1.36)\end{array}$ & $\begin{array}{c}2.53 \\
(1.59)\end{array}$ & $\begin{array}{c}2.18 \\
(1.47)\end{array}$ \\
\hline 6 & Malathion 50EC & $\begin{array}{c}7.34 \\
(2.70)\end{array}$ & $\begin{array}{c}3.31 \\
(1.80)\end{array}$ & $\begin{array}{c}2.99 \\
(1.71)\end{array}$ & $\begin{array}{c}3.75 \\
(1.93)\end{array}$ & $\begin{array}{c}7.34 \\
(2.67)\end{array}$ & $\begin{array}{c}3.46 \\
(1.86)\end{array}$ & $\begin{array}{c}3.13 \\
(1.76)\end{array}$ & $\begin{array}{l}4.33 \\
(2.08)\end{array}$ & $\begin{array}{c}3.49 \\
(1.86)\end{array}$ \\
\hline 7 & $\begin{array}{c}\text { Lambda cyhalothrin } \\
5 \% \text { EC }\end{array}$ & $\begin{array}{c}7.13 \\
(2.66)\end{array}$ & $\begin{array}{c}2.86 \\
(1.59)\end{array}$ & $\begin{array}{c}2.23 \\
(1.43)\end{array}$ & $\begin{array}{c}3.46 \\
(1.77)\end{array}$ & $\begin{array}{c}7.13 \\
(2.65)\end{array}$ & $\begin{array}{c}3.13 \\
(1.77)\end{array}$ & $\begin{array}{c}2.83 \\
(1.68)\end{array}$ & $\begin{array}{c}3.69 \\
(1.88)\end{array}$ & $\begin{array}{c}3.03 \\
(1.60)\end{array}$ \\
\hline 8 & Untreated & $\begin{array}{c}6.93 \\
(2.63)\end{array}$ & $\begin{array}{l}7.26 \\
(2.69)\end{array}$ & $\begin{array}{c}7.94 \\
(2.81)\end{array}$ & $\begin{array}{c}8.54 \\
(2.92)\end{array}$ & $\begin{array}{c}9.94 \\
(3.14)\end{array}$ & $\begin{array}{l}10.40 \\
(3.22)\end{array}$ & $\begin{array}{l}11.67 \\
(3.41)\end{array}$ & $\begin{array}{l}12.54 \\
(3.54)\end{array}$ & $\begin{array}{c}9.72 \\
(3.11)\end{array}$ \\
\hline & S. Ed. $( \pm)$ & 0.23 & 0.02 & 0.04 & $\mathbf{0 . 0 3}$ & 0.23 & 0.04 & 0.03 & 0.02 & - \\
\hline & D. $(P=0.05)$ & NS & 0.06 & 0.14 & 0.08 & NS & 0.11 & 0.08 & 0.07 & - \\
\hline
\end{tabular}

Table.2 Efficacy of selected insecticides on jassid (Amrasca biguttula biguttla) in okra

\begin{tabular}{|c|c|c|c|c|c|c|c|c|c|c|}
\hline \multirow{2}{*}{$\begin{array}{l}\text { Sl. } \\
\text { No. }\end{array}$} & \multirow{2}{*}{ Treatments } & \multicolumn{4}{|c|}{ First spray } & \multicolumn{4}{|c|}{ Second spray } & \multirow{2}{*}{$\begin{array}{c}\text { Overall } \\
\text { Mean }\end{array}$} \\
\hline & & $1 \mathrm{DBS}$ & 3 DAS & 7 DAS & 14 DAS & $1 \mathrm{DBS}$ & 3 DAS & 7 DAS & 14 DAS & \\
\hline 1 & $\begin{array}{c}\text { Thiamethoxam } \\
\text { 25\%WG }\end{array}$ & $\begin{array}{c}6.94 \\
(2.62)\end{array}$ & $\begin{array}{c}1.45 \\
(1.21)\end{array}$ & $\begin{array}{c}1.26 \\
(1.12)\end{array}$ & $\begin{array}{c}1.85 \\
(1.36)\end{array}$ & $\begin{array}{c}5.13 \\
(2.24)\end{array}$ & $\begin{array}{c}1.26 \\
(1.12)\end{array}$ & $\begin{array}{c}1.13 \\
(1.06)\end{array}$ & $\begin{array}{c}1.64 \\
(1.29)\end{array}$ & $\begin{array}{c}1.43 \\
(1.20)\end{array}$ \\
\hline 2 & Azadiractin5\% EC & $\begin{array}{c}6.93 \\
(2.63)\end{array}$ & $\begin{array}{c}3.66 \\
(1.91)\end{array}$ & $\begin{array}{c}3.14 \\
(1.76)\end{array}$ & $\begin{array}{c}4.20 \\
(2.204)\end{array}$ & $\begin{array}{c}7.54 \\
(2.69)\end{array}$ & $\begin{array}{c}3.94 \\
(1.98)\end{array}$ & $\begin{array}{c}4.26 \\
(2.06)\end{array}$ & $\begin{array}{c}4.93 \\
(2.22)\end{array}$ & $\begin{array}{c}4.01 \\
(2.00)\end{array}$ \\
\hline 3 & $\begin{array}{c}\text { Imidacloprid } \\
17.8 \% \mathrm{SL}\end{array}$ & $\begin{array}{c}6.80 \\
(2.60)\end{array}$ & $\begin{array}{c}1.26 \\
(1.12)\end{array}$ & $\begin{array}{c}1.13 \\
(1.06)\end{array}$ & $\begin{array}{c}1.73 \\
(1.31)\end{array}$ & $\begin{array}{c}5.26 \\
(2.25)\end{array}$ & $\begin{array}{c}1.13 \\
(1.06)\end{array}$ & $\begin{array}{c}0.86 \\
(0.93)\end{array}$ & $\begin{array}{c}1.46 \\
(1.21)\end{array}$ & $\begin{array}{c}1.26 \\
(1.12)\end{array}$ \\
\hline 4 & Fipronil 5\% SC & $\begin{array}{c}6.86 \\
(2.18)\end{array}$ & $\begin{array}{c}1.72 \\
(1.31)\end{array}$ & $\begin{array}{c}1.29 \\
(1.12)\end{array}$ & $\begin{array}{c}3.32 \\
(1.82)\end{array}$ & $\begin{array}{c}6.26 \\
(2.48)\end{array}$ & $\begin{array}{c}1.74 \\
(1.31)\end{array}$ & $\begin{array}{c}1.46 \\
(1.21)\end{array}$ & $\begin{array}{c}2.26 \\
(1.50)\end{array}$ & $\begin{array}{c}1.96 \\
(1.40)\end{array}$ \\
\hline 5 & Dimethoate 30EC & $\begin{array}{c}6.93 \\
(2.62)\end{array}$ & $\begin{array}{c}1.85 \\
(1.36)\end{array}$ & $\begin{array}{c}1.33 \\
(1.15)\end{array}$ & $\begin{array}{c}3.80 \\
(1.94)\end{array}$ & $\begin{array}{c}6.33 \\
(2.49)\end{array}$ & $\begin{array}{c}1.94 \\
(1.39)\end{array}$ & $\begin{array}{c}1.53 \\
(1.23)\end{array}$ & $\begin{array}{c}2.23 \\
(1.52)\end{array}$ & $\begin{array}{c}2.11 \\
(1.46)\end{array}$ \\
\hline 6 & Malathion 50EC & $\begin{array}{c}6.53 \\
(2.55)\end{array}$ & $\begin{array}{c}2.86 \\
(1.69)\end{array}$ & $\begin{array}{c}2.34 \\
(1.52)\end{array}$ & $\begin{array}{c}4.13 \\
(2.03)\end{array}$ & $\begin{array}{l}7.26 \\
(2.65)\end{array}$ & $\begin{array}{c}3.66 \\
(1.91)\end{array}$ & $\begin{array}{c}3.46 \\
(1.86)\end{array}$ & $\begin{array}{c}4.65 \\
(2.16)\end{array}$ & $\begin{array}{c}3.52 \\
(1.87)\end{array}$ \\
\hline 7 & $\begin{array}{c}\text { Lambda cyhalothrin } \\
5 \% \mathrm{EC}\end{array}$ & $\begin{array}{c}6.86 \\
(2.61)\end{array}$ & $\begin{array}{c}2.51 \\
(1.59)\end{array}$ & $\begin{array}{c}2.23 \\
(1.50)\end{array}$ & $\begin{array}{c}3.74 \\
(1.93)\end{array}$ & $\begin{array}{c}6.94 \\
(2.59)\end{array}$ & $\begin{array}{c}3.06 \\
(1.75)\end{array}$ & $\begin{array}{c}2.94 \\
(1.71)\end{array}$ & $\begin{array}{c}3.53 \\
(1.88)\end{array}$ & $\begin{array}{c}2.99 \\
(1.72)\end{array}$ \\
\hline & Untreated & $\begin{array}{c}6.86 \\
(2.61)\end{array}$ & $\begin{array}{c}7.46 \\
(2.73)\end{array}$ & $\begin{array}{c}7.93 \\
(2.81)\end{array}$ & $\begin{array}{c}8.60 \\
(2.93)\end{array}$ & $\begin{array}{l}10.13 \\
(3.15)\end{array}$ & $\begin{array}{c}10.66 \\
(3.26)\end{array}$ & $\begin{array}{l}11.46 \\
(3.38)\end{array}$ & $\begin{array}{l}10.86 \\
(3.29)\end{array}$ & $\begin{array}{c}9.49 \\
(3.08)\end{array}$ \\
\hline & S. Ed. ( \pm$)$ & 0.28 & $\mathbf{0 . 0 3}$ & 0.05 & $\mathbf{0 . 0 3}$ & 0.28 & 0.02 & 0.03 & 0.02 & - \\
\hline & C. D. $(P=0.05)$ & NS & 0.09 & 0.10 & 0.09 & NS & $\mathbf{0 . 0 7}$ & 0.08 & 0.07 & - \\
\hline
\end{tabular}

Among all the treatments Imidaclporid (1.26jassid /3leaves) recorded lowest population followed by Thiamethoxam (1.43 jasssid/3leaves), followed by next effective treatment Fipronil (1.96 jasssid /3leaves),
Dimethote (2.11 jasssid/3leaves), followed by treatment lambda Cyhalothrin (2.99jasssid /3leaves), Malathion (3.52jasssid /3leaves), while Azadirachtin (4.01jasssid /3leaves) is least effective among all the treatments. 
These findings are in accordance with the findings of Preetha et al., (2009) reported that imidacloprid was found effective against jassids and whiteflies, the other neonicotinoid, thiomethoxam also provided similar levels of protection as that of imidacloprid. Similarly, Begum and patil (2016) reported that imidacloprid 17.8SL was the most effiective treatment indicating reduction in population of leafhoppers and whitefly.Pawar et al., (2016) reported that, mean population of aphids, jassids, and whiteflies after three sprays revealed that imidaclopridwas effective and superior, the next best were thiomethoxam. Dabhi et al., (2016) reported that imidacloprid and dimethoate found to be effective against leafhopper $(81 \%)$ infesting okra.

From the critical analysis of the present findings it can be concluded that insecticides like imidacloprid (1.33whiteflies/3leaves), Thiamethoxam (1.46whiteflies/3leaves) are the best suitable for whiteflies and Imidacloprid (1.26jassid/3leaves),

Thiamethoxam (1.43jassid/3leaves) for jassid are the best suitable insecticides among other group of insecticides can be suitably incorporated in pest management against sucking pest like whitefly and jassid.

\section{Acknowledgement}

We are grateful to Dean, Head, Department of Entomology and Director of Research, Sam Higginbottom University of Agriculture, Technology and Sciences, Allahabad, U.P. for providing facilities for this research work.

\section{References}

Atwal, S.N., 1994. Agricultural pests of India and South-East Asia. Kalyani Publishers, New Delhi, India, pp. 529.
Begum Khaja Rumana and Patil S., 2016. Evaluation of Newer Molecules of Insecticides Against Sucking Pests complex Infesting Okra. Indian journal of applied research, Vol: 6 .

Dabhi, M.V., and Koshiya, D.J. 2014. Effect of abiotic factors on population dynamics of leafhopper, Amrasca biguttula biguttula (Ishida) in okra. Advance research journal of crop improvement, volume5 issue 1:1114.

Lal, O.P., and Sinha, S.R. 2005. Effect of Imidaclopril seed treatment along with some insecticidal sprayings against insectpest of Okra, Indian Journal of Entomology, 67: 328-333.

Pawar, S.A., Zanwar, P.R., Lokare, S.G., Dongarjal, R.P. and Sonkamble, M.M. 2016. Efficacy of newer insecticides against sucking pests of okra. Indian Journal of Entomology, 78(3): 257-259.

Preetha, G., Manoharan, T., Stanley, T. and Kuttalam, S. 2009. Evaluation of imidacloprid against okra jassid, Amrasca biguttula biguttula (ishida) and whitefly, Bemisia tabaci (gennadius). Indian Journal of Enfornoiogv, 71(3). 209-214.

Salim, M., 1999. Diversity: role in integrated pest management. Sci Tech. Dev., 18: 26-31.

Shinde, B.D., M.B. Sarkate., P.W. Nemade and Y.R. Sable, 2007, Bioefficacy of botanical, microbial and synthetic insecticides against okra fruit borer, Pestology, 31(3): 19-22.

Singh S., Choudhary D.P., Sharma H.C., Mahla R.S., Mathur Y.S. and Ahuja D.B. 2008. Effect of insecticidal modules against jassid and shoot and fruit borer in okra. Indian J. Entomol. 70(3):197-199.

Suraj Sarkar, Sandip Patra and Samanta, A., 2016. Efficacy of different bio-pesticides against sucking pests of okra (Abelmoschus esculentus L. Moench). Journal of Applied and Natural Science, 8 (1): 333 - 339.

\section{How to cite this article:}

Prem Kumar, K.N. and Ashwani Kumar. 2017. Efficacy of Selected Insecticides against Sucking Insect Pests [Amrasca biguttula biguttula (Ishida) and Bemisia tabaci (Gennadius)] of Okra [Abelmoschus esculentus (L.) Moench]. Int.J.Curr.Microbiol.App.Sci. 6(8): 3256-3259.

doi: https://doi.org/10.20546/ijcmas.2017.608.388 